African Crop Science Journal by African Crop Science Society is licensed under a Creative Commons Attribution 3.0 Uganda License. Based on a work at www.ajol.info/ and www.bioline.org.br/cs DOI: https://dx.doi.org/10.4314/acsj.v28i2.2

\title{
EFFICACITÉ DU BAUME DE CAJOU ET DES HUILES VÉGÉTALES POUR LA LUTTE CONTRE LES POPULATIONS DE Plutella xylostella L. 1758 (LEPIDOPTERA: PLUTELLIDAE)
}

\author{
F.A. SOTONDJI, O.K. DOURO KPINDOUI ${ }^{1}$, A.C. DJIHINTO ${ }^{2}$, E.A. DANNON ${ }^{3}$, G. ZODOME ${ }^{4}$,

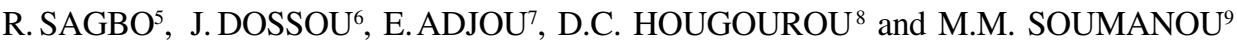 \\ Faculté des Sciences et Techniques, Université d'Abomey-Calavi, Bénin \\ ${ }^{1}$ Chargé de Recherche (CAMES) Chercheur entomologiste à IITA, Bénin \\ ${ }^{2}$ Maître de Recherche (CAMES) à l'Institut National des Recherches Agricoles du Bénin \\ ${ }^{3}$ Enseignant Chercheur en Entomologie Agricole, Université Nationale des Sciences, Technologies, \\ Ingénieries, et de Mathématiques d'Abomey (UNSTIM), Bénin \\ ${ }^{4}$ Faculté des lettres Arts et Sciences Humaines, Université d'Abomey-Calavi (FLASH), Bénin \\ ${ }^{5}$ Faculty of Science and Technology of the University of Abomey-Calavi, Bénin \\ ${ }^{6}$ Laboratoire de Bioingénierie des Procédés Alimentaires Faculté des Sciences Agronomiques \\ (FSA), Université d'Abomey-Calavi (UAC), Bénin \\ ${ }^{7}$ Enseignant- Chercheur en Technologie Alimentaire à l'Ecole Polytechnique d'Abomey-Calavi \\ (EPAC), Bénin \\ ${ }^{8}$ Enseignant-Chercheur en Entomologie et Protection des Végétaux à l'Ecole Polytechnique \\ d'Abomey-Calavi (EPAC), Bénin \\ ${ }^{9}$ Enseignant-Chercheur en Biochimie à l'Ecole Polytechnique d'Abomey-Calavi (EPAC), Bénin \\ Corresponding author: fernandsotondji@yahoo.com
}

(Received 28 May 2020; accepted 26 June 2020)

\section{RÉSUMÉ}

Le chou, Brassica oleracea L. (Capparales: Brassicaceae), est l'une des cultures les plus attaquées par un complexe de ravageurs dont Plutella xylostella L. (Lepidoptera: Plutellidea). L'objectif de cette étude était d'évaluer l'efficacité des huiles végétales et de la noix de cajou pour le contrôle de $P$. xylostella dans la production de chou. Une étude a été menée dans le laboratoire de l'institut International d'Agriculture Tropicale au Bénin. Les traitements comprenaient des huiles de trois plantes, à savoir Tephrosia purpurea, Ricinus communis, Thevetia neriifolia et la noix de cajou par rapport à un insecticide synthétique (Lambda cyhalothrine) et à celui d'un pesticide botanique (Topbio). Deux expériences ont été mises en place dans cette étude. Dans la première expérience, les produits ont été testés sur le stade L3 de P. xylostella, avec les doses de 100, 75, 50, 25 et 10\%; tandis que dans le second, les mêmes produits ont été testés sur le stade L2 du même insecte avec les doses de 50, 25, 10, 5, 3, 2 et 1\%, par rapport au témoin de référence, Lambda cyhalothrine aux doses de 10, $5,3,2,1$ et $0,5 \%$. Les variables mesurées étaient le taux de mortalité, la formation de pupes et l'émergence d'adultes de P. xylostella. Les DL 50 ont été déterminées selon la méthode de régression de Cox. Les résultats ont montré que pour tous les pesticides botaniques utilisés, quel que soit le stade larvaire de 
P. xylostella, l'effet / dose-réponse était significatif, mais moins efficaces par rapport au témoin de référence Lambda cyhalothrine. La noix de cajou et les trois huiles végétales peuvent efficacement servir d'alternatives aux pesticides de synthèse (Lambda cyalothrine) contre Plutella xylostella dans la production de chou.

Mots Clés: Brassica oleracea, chou, Ricinus communis, Tephrosia purpurea

\begin{abstract}
Cabbage, Brassica oleracea L. (Capparales: Brassicaceae), is one of the crops most attacked by a complex of pests, including Plutella xylostella L. (Lepidoptera: Plutellidea). The objective of this study was to assess the effectiveness of vegetable oils and cashews for the control of P. xylostella in cabbage production. A study was carried out in the laboratory of the International Institute of Tropical Agriculture (IITA) in Benin Treatments included oils from three plants, namely Tephrosia purpurea, Ricinus communis, Thevetia neriifolia and cashew, versus a synthetic insecticide (Lambda cyhalothrin) and a botanical pesticide (Topbio). Two experiments were set for this study. In the first experiment, the products were tested on the L3 stage of P. xylostella, with the doses of 100, 75, 50, 25 and 10\%; while in the second, the same products were tested on the L2 stage of the same insect with the doses of 50, $25,10,5,3,2$ and 1\%. All these were compared with the reference control Lambda cyhalothrin at the doses of $10,5,3,2,1$ and $0.5 \%$. The variables measured were the mortality rate, pupae formation and the emergence of adults of $P$. xylostella. The LD 50s were determined using the Cox regression method. The results showed that for all the botanical pesticides used, whatever the larval stage of $P$. xylostella was, the effect dose-response was significant, but less effective compared to the reference control Lambda cyhalothrin. Cashew and the three vegetable oils can effectively serve as alternatives to synthetic pesticides (Lambda cyhalothrin) against Plutella xylostella in cabbage production.
\end{abstract}

Key Words: Brassica oleracea, chou, Ricinus communis, Tephrosia purpurea

\section{INTRODUCTION}

Le chou, Brassica oleracea L. (Capparales: Brassicaceae), est l'une des cultures les plus attaquées par un complexe de ravageurs dont Plutella xylostella L. (Lepidoptera: Plutellidea) James et al. (2005). La forte pression parasitaire exercée par les insectes et les pathogènes sur les cultures est un obstacle majeur à l'augmentation de la productivité et à l'amélioration de la qualité de la production de chou en Afrique subsaharienne (ASS). Le papillon du chou, $P$. xylostella (L.) (Lepidoptera: Plutellidae), à lui seul, provoque des pertes pouvant atteindre plus de $90 \%$ (Verkerk et Wright, 1996; Sarfraz et al., 2005; Mondédji, 2010). C'est une espèce oligophage qui se nourrit exclusivement de plantes de la famille des Brassicacées (par exemple les crucifères). Le ravageur est attiré par les composés soufrés appelés glucosinolates, caractéristiques de cette famille de plantes. Il vit principalement sur le chou et d'autres brassicacées cultivées (moutarde, colza, navet, etc.), mais on le trouve également sur des espèces sauvages (bourse de berger, cardamine, ravenelle, etc.) qui peuvent servir de réservoir pendant les périodes où les cultures sont non disponibles (Muhamad et al. 1994). Ces composés chimiques sont des phagostimulants pour les chenilles et des stimulants de ponte chez les femelles (Gupta et Thornsteinson, 1998).

La méthode de lutte la plus courante contre ce ravageur est l'utilisation abusive de pesticides de synthèse (Dovlo, 2007). Cependant, ces méthodes de contrôle ont montré certaines limites: par exemple: dégradation de la biodiversité, y compris l'élimination des ennemis naturels (Fur-long 
et al., 2012); impact sur la santé des utilisateurs et des consommateurs (résidus de pesticides), augmentation des coûts de production (Chilcutt et Tabashnik, 1997); et apparition de souches résistantes (Hooks and Johnson, 2003; Macharia et al., 2005; Sarfraz et al., 2005; Shelton et al., 2007; Huang et al., 2010).

Une étude diagnostique des problèmes phytosanitaires réalisée au Bénin sur la protection des légumes dans le sud du Bénin a montré que le chou est l'une des cultures nécessitant une variété et des doses élevées de pesticides chimiques dangereux dans l'agroécosystème urbain et périurbain (James et al., 2005). De plus, la résistance de $P$. xylostella aux pesticides est répandue (Guilloux, 2000). Plutella xylostella a montré une résistance à presque tous les insecticides agricoles et même au bio-pesticide basé sur la toxine de Bacillus thuringiensis (Bt) (Tabashnik et al., 1990; Liu et al., 1995). Le développement de nouvelles méthodes de phytoprotection à moindre impact écologique constitue une alternative potentielle. Parmi les nouvelles technologies de protection des cultures, l'utilisation des insecticides botaniques efficaces et moins toxiques serait une alternative à l'utilisation des pesticides de synthèse dans le contrôle des insectes ravageurs Philogène et al. (2003). L'objectif de cette étude était d'évaluer l'efficacité des huiles végétales et de la noix de cajou pour le contrôle de $P$. xylostella au laboratoire dans la production de chou.

\section{MATÉRIELS ET MÉTHODES}

Milieu d'étude. Les expériences ont été réalisées dans les laboratoires de pathologie de l'IITA (Bénin) et dans le Laboratoire de recherche en biologie appliquée (LARBA) de l'Université d'Abomey-Calavi (UAC), Bénin, d'Avril à Septembre 2019. Les conditions ambiantes dans les laboratoires étaient de 26 $\pm 0,5^{\circ} \mathrm{C}$ et $65,5 \pm 5 \% \mathrm{HR}$.

Insectes pest. Les larves de $P$. xylostella utilisées ont été fournies par le Laboratoire d'élevage de l'IITA (Bénin). Ce sont des insectes récoltés sur les sites maraîchers de Sèmé Kpodji et de Houéyiho. Les larves ont été nourries avec les feuilles de la plante hôte (Brassica oleracea var. Capitata).

Expériences. L'appétit des larves pour différentes doses de pesticides botaniques, provenant du témoin de référence (Lambda) a été testé. Pour la première expérience, les doses d'extraits botaniques et du témoin de référence étaient de 100, 75, 50, 25 et 10\%. Celles de la deuxième expérience de $50,25,10,5,3$ et $1 \%$ pour les huiles végétales et $10 ; 5,3,2$, 1 et $0,5 \%$ pour le témoin référencé (Lambda). Des larves de stade 2 et 3 de $P$. xylostella ont été utilisées. De l'eau distillée, sans huile ni Lambda, a été utilisée pour traiter le contrôle négatif. L'appétence a été évaluée en estimant la surface foliaire consommée par les larves après 24 heures. Chaque disque de feuille de chou caillé de rayons $2 \mathrm{~cm}$ a été placé chacun en présence des larves et a été retiré après 24 heures; avant d'être placé sur du papier millimétré. Les surfaces consommées ont été reproduites sur ce papier puis évaluées.

Traitements des données. Pour déterminer l'effet des différents extraits sur les larves des stades 2 et 3 de $P$. xylostella, cinq pesticides botaniques et un pesticide chimique (Lambda cyalothrin) ont été utilisés à différentes doses. Pour la première expérience, cinq doses ont été préparées comme suit: 100, 75, 50, 25 et $10 \%$ de chacun des extraits botaniques et le produit chimique synthétique ont été préparés avec $30 \mathrm{mg}$ de lécithine plus du savon sans détergent, dans l'eau distillée pour chaque solution mère. L'inscticide Lambda a été utilisé dans la même proportion que les autres extraits dans la première expérience sur les stades L2. Pour la deuxième expérience, les doses d'application des pesticides botaniques utilisés étaient: $50,25,10,5,3$ et $1 \%$; et $10,5,3,2,1$ et $0,5 \%$ pour le contrôle de référence (Lambda) sur les larves du stade 2. Le contrôle négatif était à nouveau de l'eau distillée. Un 
agent mouillant, tween80, a été ajouté aux différents traitements.

Tests de toxicité biologique. Le test de toxicité biologique était une méthode de trempage des feuilles similaire à celles utilisées par Tabashnik et al. (1990). Pour l'expérience de test de toxicité biologique, des disques de feuilles de chou traités ont été donnés à des larves de deuxième et troisième stades. Ainsi, du tissu foliaire ( $6 \mathrm{~cm}$ de diamètre) a été coupé à partir de plants de chou non infestés produits en serre. Ces feuilles ont été lavées avec 10\% d'eau de Javel, puis rincées à l'eau du robinet. Les feuilles individuelles ont été immergées dans la solution d'insecticide préparée pendant 30 secondes et suspendues verticalement pour être séchées à température ambiante pendant 5 min. Dix larves de P. xylostella en élevage ont ensuite été placées sur la face supérieure d'un disque foliaire de $6 \mathrm{~cm}$ de diamètre découpé placé dans chaque boîte de Pétri (6 $\mathrm{cm} \times 1,5 \mathrm{~cm}$ ) avec un couvercle perforé, recouvert de mousseline pour l'aération.

Les disques de feuilles ont été placés sur du coton imbibé d'eau dans des boîtes de Pétri de $8,5 \mathrm{~cm}$ de diamètre pour maintenir l'humidité des feuilles. Chacune des concentrations a été répétée trois fois dans un bloc complètement aléatoire. Ainsi, 180 larves L2 et L3 testées par concentration. Le traitement témoin consistait en 60 larves L2 et 60 larves L3 de P. xylostella traitées avec une solution de tween 80 stérilisée. Les boîtes de Pétri ont ensuite été placées dans des plateaux en laboratoire à une température variant entre $26 \pm 1{ }^{\circ} \mathrm{C}$.

Données collectées. La mortalité larvaire a été enregistrée toutes les 24 heures, jusqu'au neuvième jour après le traitement. Les larves étaient considérées comme mortes lorsqu'elles ne bougeaient pas, lorsqu'elles étaient légèrement pincées avec une pince (Hill et Foster, 2000). Les disques foliaires ont été changés tous les trois jours avec des feuilles fraîches et non traitées. Les surfaces foliaires ont été calculées à 24 heures pour évaluer l'effet de la magnétisation sur le contrôle des larves.

L'analyse des données. Les taux de mortalité moyens des larves, des pupes et de l'émergence des pupes obtenus à la fin des observations ont été utilisés pour faire les tableaux. Les taux de mortalité des insectes traités ont été corrigés à l'aide de la formule d'Abbott (1925):

$$
\begin{aligned}
& \% \mathrm{Mc}=(\mathrm{Me}(\%)-\mathrm{Mt}(\%)) /(100-\mathrm{Mt}(\%)) \\
& \mathrm{x} 100
\end{aligned}
$$

Où: $\mathrm{Mc}=$ mortalité corrigée en pourcentage, $\mathrm{Me}=$ mortalité de l'échantillon testé et $\mathrm{Mt}=$ mortalité dans le contrôle non traité

Le taux d'émergence a été calculé en fonction du nombre de pupes formées dans chaque traitement. Après une transformation arc-sinus, une analyse de la variance a été effectuée. Lorsque l'ANOVA a révélé des différences significatives entre les traitements, le test SNK (Student-Newman Keuls), au seuil de 5\%, a été réalisé afin de séparer les moyennes des différents traitements. Pour toutes ces analyses, le logiciel XLSTAT version 2019 a été utilisé.

DL50 estimée (dose létale 50). Les données temps-dose-mortalité ont été analysées et modélisées à l'aide du modèle de "régression de Cox" (SPSS, 1989-2007). Les modèles de régression de Cox utilisent la fonction de risque pour estimer le risque d'échec relatif. La fonction de risque, $h(t)$ est une évaluation du décès potentiel d'un individu par unité de temps à un moment donné, puisque l'individu a survécu jusqu'à ce point. Les modèles de «régression de Cox» sont exprimés en termes de fonction de risque comme suit:

$\mathrm{h}(\mathrm{t})=[\mathrm{h} 0(\mathrm{t}) \llbracket] \mathrm{e} \rrbracket \wedge((\mathrm{BX}))$

Où: $\mathrm{X}$ représente le log (dose), $\mathrm{B}$ le coefficient de régression qui est le risque relatif (ici le risque de décès instantané) associé à un traitement par rapport à un autre traitement, 
et e la base du logarithme naturel et h0 (t) est le risque fonction lorsque $\mathrm{X}$ est égal à 0 .

La fonction de risque cumulatif, $\mathrm{H}(\mathrm{t})$, est liée à la fonction de survie et peut être dérivée de la fonction de survie comme suit:

$H(t)=\ln S(t)$

La fonction de risque et la fonction de survie sont étroitement liées, et toutes deux ont été calculées à l'aide de la méthode de «régression de Cox»(SPSS, 1989-2007). La DL50 est définie comme la dose d'un agent (chimique ou biologique) nécessaire pour provoquer la mort de la moitié des organismes testés à un moment donné après l'application (Maddox, 1982). La DL50 peut être dérivée de l'équation comme suit:

$\mathrm{X}=\left\langle 10>^{\wedge}\left(\ln ^{10}\left(\ln ^{10}(0,5)-\ln ^{10}(\mathrm{t})\right)\right) / \mathrm{BX}\right)$

Les intervalles de confiance pour la DL50 ont été calculés sur la base des mêmes équations, en utilisant les informations suivantes: écart type (SE) de B et écart type (SE) de h0 (t).

\section{RÉSULTATS}

Appétence des larves L2 et L3. Le Tableau 1 montre que les surfaces moyennes consommées par une larve L3 de $P$. xylostella sont comprises entre $21,33 \pm 3,519$, et 268,44 $\pm 21,51 \mathrm{~mm}^{2}$ à un taux d'application de $10 \%$ respectivement pour l'huile Tephrosia et le traitement témoin (0\%). Les surfaces moyennes consommées par une larve L2 de P. xylostella sont comprises entre $67,80 \pm$ $31,22,40,31 \pm 74,94$ et $205,44 \pm 33,54 \mathrm{~mm}^{2}$ à une dose d'application de $1 \%$ respectivement T. purpurea, Lambda cyalothrine et le témoin négatif (Tableau 2). Les surfaces foliaires consommées par les larves de $P$. xylostella étaient plus importantes au niveau des feuilles non traitées qu'au niveau des feuilles traitées.

Le test de comportement alimentaire des larves a montré que la consommation des feuilles dépend de la dose de pesticide chimique botanique ou synthétique utilisée. Plus la dose 
est élevée, moins la consommation a été observée pour les extraits de plantes et l'insecticide chimique synthétique. Les plus grandes surfaces foliaires ont été consommées chez les larves L3 à des doses de 10\% des biopesticides testés, tandis qu'à partir de cette dose, un manque de palatabilité a été observé chez les larves L2 avec les mêmes produits (Tableau 2).

Il y avait une différence significative entre les zones consommées par les larves L2 de $P$. xylostella $(\mathrm{F}=605,83 ; \mathrm{df}=41 ; \mathrm{P}<0,0001)$ au niveau des témoins que les produits utilisés. Pour les doses de 100, 75, 50, d'extraits d'huile végétale et de CNSL $25 \%$, un phénomène d'inappétence a également été constaté chez certaines larves L3 qui, malgré le jeûne avant le test, ne consommaient aucune partie de la feuille. Il y avait également une différence significative entre les zones consommées par les larves L3 de P. xylostella au niveau des témoins non traités et les larves traitées $(\mathrm{F}=505,836 ;$ dof $=41 ; \mathrm{P}<0,0001)$.

Effets comparés des huiles végétales sur le taux de mortalité des larves L2 et L3, la formation des chrysalides et l'émergence des adultes de $P$. xylostella. Après avoir exposé les larves du stade 3 de l'espèce $P$. xylostella aux différentes concentrations des trois huiles végétales, du baume de cajou, du Topbio et de l'insecticide de synthèse Lambda pendant 09 jours, le taux de mortalité et d'émergence varient selon les doses (Tableau 3). Pour tous les extraits, la mortalité des larves a atteint un taux de $100 \%$ à partir d'une concentration de $25 \%$. En ce qui concerne, les deux témoins de référence Lambda et Topbio la mortalité est plafonnée à $100 \%$ dès la concentration de $10 \%$. Il existe une différence significative entre les larves traitées à une dose d'application de $1 \%$ et le témoin non traité. Il existe également une différence significative entre les plus fortes doses (100, 75,50 et $25 \%$ ) et les doses les plus faibles $(10,5,3$ et $1 \%)$. De l'ensemble de ces résultats un premier classement de l'efficacité toxique des extraits testés est mis en évidence, ainsi 
les extraits les plus toxiques sont ceux des $T$. purpurea, CNSL, et $R$. communis, et le moins toxique celui de $T$. neriifolia. Une analyse comparative de la mortalité larvaire induite par les différents extraits des pesticides botaniques montre une plus grande efficacité des huiles de Tephrosia et du CNSL $(\mathrm{p}<0.05)$, même à une dose de $5 \%$ de la solution usitée, le taux de mortalité va au-delà de $60 \%$ après application sur les larves L2 (Tableau 4). Des mortalités intermédiaires sont induites par les huiles végétales de $R$. communis et de $T$. neriifolia. Cependant, elle provoque la mortalité larvaire la plus faible. Le temps d'exposition varie aussi en fonction du pesticide et de la dose utilisé pour les différents traitements. Les résultats d'analyse de la variance relatifs à l'effet insecticide des huiles de plantes sur $P$. xylostella révèlent que le taux de mortalité a une variation très hautement significative suivant la dose, le stade larvaire et le temps d'exposition considéré d'une part, selon la plante, le stade larvaire et le temps d'exposition d'autre part $(\mathrm{P}<0,001)$. Cela implique que l'effet insecticide observé dépend de la nature de la plante, de la dose et du stade larvaire (Tableaux 3 et 4).

Effet des traitements sur la formation des chrysalides et le taux d'émergence des adultes de $P$. xylostella issus des larves $\mathrm{L} 2$ et L3 traitées. Le Tableau 5, montre que les taux de chrysalidation de $P$. xylostella par rapport aux larves survivantes après $24 \mathrm{~h}$ d'exposition aux feuilles de chou traitées étaient plus faibles pour les doses d'application de $10 \%$ de CNSL, de $T$. purpurea, $R$. communis de $T$. neriifolia, et du Topbio $(18,7,17,77$, $28,4,30$, et $14,15 \%$ de chrysalides respectivement) sur les larves L3 comparé aux taux de chrysalidation obtenus des doses de $1 \%$ des mêmes produits sur les larves L2 $(44,12,33,66,52,45,62,14$ et $42,77 \%$ respectivement). Les taux d'émergence des individus ayant survécu aux différents traitements ont varié de 50,2\% à 77,8\% à une dose d'application de $10 \%$ sur les larves L3 et de 75,42 à 94,10\% à une dose d'application 


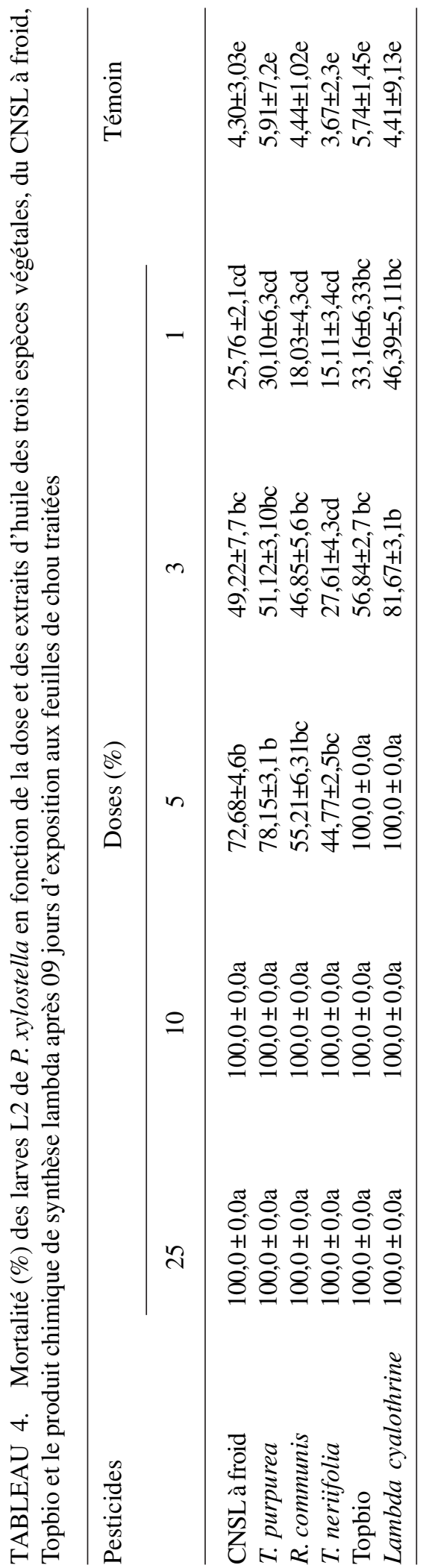

F.A. SOTONDJI et al.

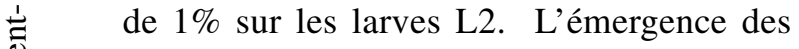
femelles était supérieure à celle des mâles. Les témoins ont enregistré le plus fort taux d'émergence des adultes que les larves traitées.

DL50 en fonction du temps des pesticides utilisés sur les larves $L 2$ et $L 3$ de $P$. xylostella. Les valeurs de DL50 et les intervalles de confiances pour chaque pesticide botanique sont présentés dans les Tableaux 8 et 9 . De ces résultats, il ressort que la mortalité était dépendante de la dose et du temps après exposition des larves L2 et L3 aux différents pesticides. Pour une durée d'observation de 05 jours, la DL50 du Topbio était de $3 \%$ contre $12,5 \%$ pour 02 jours et $5,1 \%$ pour 04 jours alors que les DL50 du témoin de référence Lambda cyalothrine étaient de 1,2 et de 1,9\% respectivement sur les larves L2 et L3 pour 02 jours et 05 jours. Les DL50 obtenues avec l'huile de $T$. purpurea, le CNSL, l'huile de ricinus et de Thevetia étaient également dépendantes de la dose et du temps. De, 13,4, 13,8 , 15,6, et16,9\% respectivement pour l'huile de T. purpurea, CNSL, Ricinus et T. neriifolia aux 02 jours sur les larves L3. La DL50 pour ces mêmes pesticides botaniques pour le contrôle des larves L2 de P. xylostella est passé à $8,6,8,1,9,3,14$, et de $15,5 \%$ au bout de ( 2 jours) alors qu'il fallait $1,4,2,2,4,2,8$ et $3,1 \%$ pour contrôler $50 \%$ de ces larves en 120 heures (5 jours) respectivement pour Topbio, Tephrosia, CNSL, Ricinus et Thevetia. Considérant les DL50 et les intervalles de confiances aux différents temps après traitement, pour un même temps, la DL50 de Thevetia a été plus élevée que celles des autres. Topbio a été plus efficace que les autres extraits végétaux. Ceci se remarque également par la diminution de la largeur des intervalles de confiance, comparativement aux celles des intervalles de confiance de $T$. purpurea, CNSL, $R$. communis et $T$. neriifolia. Ces valeurs montrent l'existence d'une relation dose-réponse dans le temps pour les cinq pesticides botaniques. Le témoin de référence Lambda cyalothrine a donné la valeur $B(68,287)$ la plus élevée 
TABLEAU 5. Taux moyens de chrysalidation, d'adultes de P. xylostella émergés après application de différentes doses de CNSL, Tephrosia purpurea, Ricinus communis, thevetia neriifolia, Topbio et Lambda

\begin{tabular}{|c|c|c|c|c|c|c|c|c|}
\hline Traitement & $\begin{array}{l}\text { Stades } \\
\text { larvaires }\end{array}$ & Dose $(\%)$ & $\begin{array}{c}\% \text { de } \\
\text { chrysalidation }\end{array}$ & $\begin{array}{c}\% \\
\text { émergence } \\
\text { des adultes }\end{array}$ & $\begin{array}{l}\text { Nombre de } \\
\text { mâle }\end{array}$ & $\begin{array}{l}\text { Nombre de } \\
\text { femelle }\end{array}$ & $\begin{array}{c}\text { Malformées } \\
\text { femelle }\end{array}$ & $\begin{array}{l}\text { Malformés } \\
\text { mâle }\end{array}$ \\
\hline Témoin & & 0 & 96,4 & 95,12 & 31 & 19 & 0 & 0 \\
\hline CNSL à froid & $\begin{array}{l}\mathrm{L} 3 \\
\mathrm{~L} 2\end{array}$ & $\begin{array}{r}10 \\
5 \\
3 \\
1\end{array}$ & $\begin{array}{r}18,7 \\
12,16 \\
18,44 \\
44,12\end{array}$ & $\begin{array}{r}61,4 \\
72,32 \\
78,39 \\
89,14\end{array}$ & $\begin{array}{r}1 \\
6 \\
7 \\
10\end{array}$ & $\begin{array}{l}3 \\
2 \\
4 \\
6\end{array}$ & $\begin{array}{l}1 \\
1 \\
2 \\
0\end{array}$ & $\begin{array}{l}0 \\
0 \\
1 \\
0\end{array}$ \\
\hline T. Purpurea & $\begin{array}{l}\mathrm{L} 3 \\
\mathrm{~L} 2\end{array}$ & $\begin{array}{r}10 \\
3 \\
1\end{array}$ & $\begin{array}{l}17,77 \\
15,78 \\
33,66\end{array}$ & $\begin{array}{r}50,2 \\
78,14 \\
84,04\end{array}$ & $\begin{array}{l}2 \\
7 \\
9\end{array}$ & $\begin{array}{l}2 \\
4 \\
5\end{array}$ & $\begin{array}{l}0 \\
5 \\
0\end{array}$ & $\begin{array}{l}1 \\
3 \\
1\end{array}$ \\
\hline R. communis & $\begin{array}{l}\mathrm{L} 3 \\
\mathrm{~L} 2\end{array}$ & $\begin{array}{r}10 \\
5 \\
3 \\
1\end{array}$ & $\begin{array}{r}28,4 \\
17,60 \\
40,05 \\
52,45\end{array}$ & $\begin{array}{l}67,78 \\
64,33 \\
78,84 \\
91,33\end{array}$ & $\begin{array}{r}3 \\
7 \\
9 \\
11\end{array}$ & $\begin{array}{l}4 \\
3 \\
4 \\
6\end{array}$ & $\begin{array}{l}1 \\
3 \\
3 \\
8\end{array}$ & $\begin{array}{l}0 \\
2 \\
0 \\
5\end{array}$ \\
\hline T. neriifolia & $\begin{array}{l}\mathrm{L} 3 \\
\mathrm{~L} 2\end{array}$ & $\begin{array}{r}10 \\
5 \\
3 \\
1\end{array}$ & $\begin{array}{r}30 \\
22,78 \\
50,55 \\
62,14\end{array}$ & $\begin{array}{r}77,8 \\
70,45 \\
77,14 \\
94,10\end{array}$ & $\begin{array}{r}7 \\
8 \\
10 \\
12\end{array}$ & $\begin{array}{l}5 \\
4 \\
6 \\
7\end{array}$ & $\begin{array}{l}2 \\
4 \\
3 \\
2\end{array}$ & $\begin{array}{l}1 \\
2 \\
1 \\
3\end{array}$ \\
\hline Topbio & $\mathrm{L} 2$ & $\begin{array}{l}3 \\
1\end{array}$ & $\begin{array}{r}25,9 \\
42,77\end{array}$ & $\begin{array}{l}70,18 \\
80,17\end{array}$ & $\begin{array}{r}8 \\
10\end{array}$ & $\begin{array}{l}5 \\
3\end{array}$ & $\begin{array}{l}3 \\
0\end{array}$ & $\begin{array}{l}1 \\
0\end{array}$ \\
\hline Lambda cyalothrine & $\mathrm{L} 2$ & $\begin{array}{r}3 \\
2 \\
1 \\
0,5\end{array}$ & $\begin{array}{r}8,16 \\
21,36 \\
36,31 \\
65,96\end{array}$ & $\begin{array}{l}51,02 \\
64,35 \\
75,42 \\
91,23\end{array}$ & $\begin{array}{r}4 \\
4 \\
5 \\
11\end{array}$ & $\begin{array}{l}0 \\
2 \\
4 \\
5\end{array}$ & $\begin{array}{l}1 \\
2 \\
0 \\
0\end{array}$ & $\begin{array}{l}0 \\
1 \\
3 \\
0\end{array}$ \\
\hline
\end{tabular}


TABLEAU 6. Modèle d'estimation de la valeur B résultant de la régression Cox pour les produits utilisés y inclus les coefficients de Wald

\begin{tabular}{lcccccc}
\hline Produits & Stade plutella & B & SE & Wald & ddl & Prob. \\
\hline CNSL à chaud & L3 & 5,280 & 0,838 & 35,249 & 1 & 0,000 \\
T. purpurea & L3 & 5,339 & 0,865 & 39,894 & 1 & 0,000 \\
R. communis & L3 & 4,841 & 0,814 & 32,653 & 1 & 0,000 \\
T. neriifolia & L3 & 4,802 & 0,801 & 30,216 & 1 & 0,000 \\
Topbio & L3 & 5,891 & 0,901 & 40,417 & 1 & 0,000 \\
Lambda & L3 & 6,286 & 0,922 & 46,474 & 1 & 0,000 \\
\hline
\end{tabular}

$\mathrm{B}=$ Valeur B de la régression Cox ; SE = Erreur Standard ; Wald = coefficient de Wald ; ddl = degré de liberté ; Prob. = Probabilité

TABLEAU 7. Modèle d'estimation de la valeur B résultant de la régression Cox pour les produits utilisés y inclus les coefficients de Wald

\begin{tabular}{lcccccc}
\hline Produits & Stade plutella & B & SE & Wald & ddl & Prob. \\
\hline CNSL à chaud & L2 & 13,585 & 1,037 & 171,701 & 1 & 0,000 \\
T. purpurea & L2 & 12,496 & 0,983 & 161,438 & 1 & 0,000 \\
R. communis & L2 & 11,934 & 0,952 & 157,217 & 1 & 0,000 \\
T. neriifolia & L2 & 11,113 & 0,895 & 154,308 & 1 & 0,000 \\
Topbio & L2 & 14,254 & 1,057 & 181,989 & 1 & 0,000 \\
Lambda & L2 & 68,287 & 4,713 & 209,897 & 1 & 0,000 \\
\hline
\end{tabular}

$\mathrm{B}=$ Valeur B de la régression Cox ; SE = Erreur Standard ; Wald = coefficient de Wald ; ddl = degré de liberté ; Prob. = Probabilité

(Tableau 6) et par conséquent, les valeurs de DL50 les moins élevées (Tableau 8). La relation dose-réponse a été très bonne pour Lambda cyalothrine. Parmi tous les pesticides botaniques le Topbio a donné la valeur de B $(14,254)$ la plus élevée (Tableau 6) et les valeurs de DL50 les plus faibles (Tableau 9). Les valeurs de $B$ pour tous les pesticides sont positives cela explique que la relation dose réponse a été très bonne pour tous les pesticides utilisés (Tableaux 6 et 7).

\section{DISCUSSION}

D'une manière générale, les résultats obtenus des différentes expérimentations au laboratoire montrent que les pesticides botaniques à base de T. purpurea, $R$. communis, T. neriifolia et du baume de cajou extraction à froid utilisés sont toxiques aux larves de $P$. xylostella. Ces résultats corroborent les études sur l'effet larvicides de différents extraits à base de plantes par (Kétho et al., 2002 ; Sanda et al., 2006 ; Agboka et al., 2009). Les différents résultats obtenus montrent également que la mortalité des larves de P. xylostella est fonction de la dose qu'elles reçoivent. Il ressort des résultats que les mortalités moyennes cumulées augmentent avec les concentrations des différents pesticides.

L'étude des effets des pesticides botaniques sur le développement et le comportement alimentaire de $P$. xylostella a montré que tous les traitements ont eu un impact significatif 
TABLEAU 8. DL50 en fonction du temps des pesticides utilisés sur les larves L3 de P. Xylostella

\begin{tabular}{|c|c|c|c|c|}
\hline Produits & $\begin{array}{l}\text { Temps après } \\
\text { traitement (jours) }\end{array}$ & $\begin{array}{l}\text { DL50 } \\
\text { (\% de dilution) }\end{array}$ & $\begin{array}{c}\text { IC (Intervalles } \\
\text { de confiances) } \%\end{array}$ & $P$ (Probabilité) \\
\hline \multirow[t]{5}{*}{ CNSL à froid } & 1 & 25,4 & {$[29,4-26,2]$} & $<0,001$ \\
\hline & 2 & 13,8 & {$[16,8-10,7]$} & \\
\hline & 3 & 8,1 & {$[9,9-7,6]$} & \\
\hline & 4 & 5,8 & {$[6,6-5,5]$} & \\
\hline & 5 & 4,7 & {$[5,5-4,5]$} & \\
\hline \multirow[t]{5}{*}{ T. purpurea } & 1 & 24,1 & {$[28,5-27,0]$} & $<0,001$ \\
\hline & 2 & 13,4 & {$[16,6-10,1]$} & \\
\hline & 3 & 7,8 & {$[9,8-7,2]$} & \\
\hline & 4 & 6,0 & {$[7,2-5,7]$} & \\
\hline & 5 & 4,3 & {$[5,3-4,1]$} & \\
\hline \multirow[t]{5}{*}{ R. communis } & 1 & 27,2 & {$[33,1-32,4]$} & $<0,001$ \\
\hline & 2 & 15,6 & {$[18,5-15,3]$} & \\
\hline & 3 & 8,9 & {$[11,4-9,3]$} & \\
\hline & 4 & 6,2 & {$[8,1-7,0]$} & \\
\hline & 5 & 5,4 & {$[6,4-5,7]$} & \\
\hline \multirow[t]{5}{*}{ T. neriifolia } & 1 & 29,4 & {$[36,4-30,2]$} & $<0,001$ \\
\hline & 2 & 16,9 & {$[19,3-13,1]$} & \\
\hline & 3 & 10,1 & {$[11,4-9,0]$} & \\
\hline & 4 & 7,7 & {$[8,1-7,7]$} & \\
\hline & 5 & 6,0 & {$[7,0-6,2]$} & \\
\hline \multirow[t]{5}{*}{ TopBio } & 1 & 20,2 & {$[24,3-18,1]$} & $<0,001$ \\
\hline & 2 & 12,5 & {$[17,8-9,9]$} & \\
\hline & 3 & 7,3 & {$[9,9-7,5]$} & \\
\hline & 4 & 5,1 & {$[6,8-5,1]$} & \\
\hline & 5 & 3,0 & {$[3,2-2,4]$} & \\
\hline \multirow[t]{5}{*}{ Lambda cyalothrine } & 1 & 7,4 & {$[8,1-7,3]$} & $<0,001$ \\
\hline & 2 & 5,6 & {$[7,6-5,4]$} & \\
\hline & 3 & 3,7 & {$[4,2-3,5]$} & \\
\hline & 4 & 3,1 & {$[3,5-2,7]$} & \\
\hline & 5 & 1,9 & {$[2,6-2,1]$} & \\
\hline
\end{tabular}

sur la mortalité et l'alimentation des larves de P. xylostella. Le taux de mortalité des larves a augmenté dans le temps pour atteindre des pics en fonction des doses. L'effet larvicide des extraits de $R$. communis avait déjà été mis en exergue par (Olsnes, 2004; Kumar et al., 2007; Lui et al., 2007, Tounou A.K. et al.
2011, sur $P$. xylostella, par Aouinty et al. (2006) sur quatre espèces de moustiques, Culex pipiens (L.), Aedes caspius (Pallas), Culiseta longiareolata (Aitken) et Anopheles maculipennis (Meigen) Anani et al. (2004) et par Laghdaf et Ferji, (2005) sur les nématodes. La toxicité de la plante est due à la présence 
TABLEAU 9. DL50 en fonction du temps des pesticides utilisés sur les larves L2 de P. Xylostella

\begin{tabular}{|c|c|c|c|c|}
\hline Produits & $\begin{array}{l}\text { Temps après } \\
\text { traitement (jours) }\end{array}$ & $\begin{array}{l}\text { DL50 } \\
\text { (\% de dilution) }\end{array}$ & $\begin{array}{c}\text { IC (Intervalles } \\
\text { de confiances) } \%\end{array}$ & $P$ (Probabilité) \\
\hline \multirow[t]{5}{*}{ CNSL à froid } & 1 & 17,2 & {$[20,4-14,9]$} & $<0,001$ \\
\hline & 2 & 9,3 & {$[11,0-8,0]$} & \\
\hline & 3 & 4,2 & {$[5,0-3,6]$} & \\
\hline & 4 & 3,3 & {$[2,8-2,0]$} & \\
\hline & 5 & 2,4 & {$[2,0-1,7]$} & \\
\hline \multirow[t]{5}{*}{ T. purpurea } & 1 & 16,2 & {$[19,9-14,1]$} & $<0,001$ \\
\hline & 2 & 8,1 & {$[9,46-7,6]$} & \\
\hline & 3 & 3,7 & {$[4,31-3,2]$} & \\
\hline & 4 & 2,7 & {$[2,9-2,1]$} & \\
\hline & 5 & 2,0 & {$[1,8-1,3]$} & \\
\hline \multirow[t]{5}{*}{ R. communis } & 1 & 20,5 & {$[24,1-17,18]$} & $<0,001$ \\
\hline & 2 & 14,7 & {$[17,3-12,8]$} & \\
\hline & 3 & 8,1 & {$[9,5-7,0]$} & \\
\hline & 4 & 3,8 & {$[4,5-3,3]$} & \\
\hline & 5 & 2,8 & {$[3,7-2,7]$} & \\
\hline \multirow[t]{5}{*}{ T. neriifolia } & 1 & 21,4 & {$[25,5-18,5]$} & $<0,001$ \\
\hline & 2 & 15,5 & {$[15,5-11,3]$} & \\
\hline & 3 & 9,8 & {$[8,5-6,2]$} & \\
\hline & 4 & 4,7 & {$[4,5-3,3]$} & \\
\hline & 5 & 3,1 & {$[3,7-2,7]$} & \\
\hline \multirow[t]{5}{*}{ TopBio } & 1 & 15,9 & {$[18,8-13,8]$} & $<0,001$ \\
\hline & 2 & 8,6 & {$[10,2-7,4]$} & \\
\hline & 3 & 4,2 & {$[5,0-3,7]$} & \\
\hline & 4 & 2,1 & {$[2,5-1,8]$} & \\
\hline & 5 & 1,4 & $1,6-1,0]$ & \\
\hline \multirow[t]{5}{*}{ Lambda cyalothrine } & 1 & 4,3 & {$[5,0-3,8]$} & $<0,001$ \\
\hline & 2 & 3,1 & {$[3,6-2,8]$} & \\
\hline & 3 & 2,1 & {$[2,4-1,8]$} & \\
\hline & 4 & 1,7 & {$[1,9-1,5]$} & \\
\hline & 5 & 1,2 & {$[1,4-1,1]$} & \\
\hline
\end{tabular}

de ricine, une substance soluble dans l'eau, glycoprotéine concentrée dans la graine endosperme.

T. neriifolia est une plante à intérêt médicinal à cause de sa propriété toxique (Garima et Amla, (2011). L'activité insecticide des extraits a été prouvée par plusieurs auteurs dans le domaine agricole. En effet, les extraits des organes de la plante ont montré une activité biocide sur $C$. maculatus (Mollah et Islam, 2007) et contre les charançons adultes de maïs (Wanyika et al., 2009) et sur les moustiques Anopheles gambiae (Akpo et al., 2017). Les travaux réalisés par Chougourou et al. (2012) 
ont prouvé aussi l'efficacité biocide de l'huile extraite des amandes des graines sur les larves de Musca domestica. Des études similaires réalisées en Tanzanie ont montré aussi l'effet biocide des extraits de T. purpurea sur les larves de moustiques (Puyvelde et al., 1987; Mayunga, 2002; Touqeer et al., 2013). L'effet larvicide du CNSL a été prouvé par Akpo et al. (2017) sur A. gambiae et par Azonkpin et al 2019 sur $H$. armigera.

Le taux de mortalités a été progressif pour tous les produits en fonction de la dose, mais néanmoins, il existe une différence significative entre les différents produits testés sur les larves $(\mathrm{P}<0,001)$. Au stade L2 et L3, le taux de mortalité des larves à une dose d'application de $10 \%$ des différents pesticides botaniques utilisés et les témoins de références sont statistiquement différent. Ce taux de mortalité a été nettement supérieur sur les larves L2, lorsque ces mêmes doses ont été appliquées sur les stades L3 ; ceci est dû probablement à la taille plus élevée des larves L3, comparativement à celle des L2. Ces résultats sont similaires à ceux obtenus par Bateman $e t$ al. (1996) qui ont trouvé que l'infection des criquets par les champignons est fonction de leur poids. Au-delà de $3 \%$ de l'huile de $R$. communis, et de $T$. neriifolia ; la réponse effet/ dose n'est plus perceptible sur les larves L2, en raison probablement de la grande variabilité dans la mortalité d'une répétition à l'autre alors qu'il faut aller jusqu'à $1 \% T$. purpurea et de CNSL avant que le taux de mortalité soit proche des témoins négatifs.

L'émergence des adultes a également été en fonction à la fois du pesticide botanique utilisé et des doses testées. Les quatre pesticides botaniques et les témoins de références utilisés ont eu un effet néfaste sur la chrysalidation et par suite sur l'émergence des adultes de $P$. xylostella. On note une baisse sensible de l'émergence des adultes qui est également dépendante des doses utilisées. $\mathrm{Ce}$ constat a été précédemment fait sur $P$. xylostella chez qui les extraits de feuilles de neem ont régulé la croissance des larves en entrainant une diminution du taux d'adultes émergés (Mondedji, 2010). Il ressort que les doses de 1 et $3 \%$ des pesticides botaniques ont enregistré le plus fort taux de chrysalides et l'émergences des adultes. Les chrysalides issues des plus fortes doses des pesticides botaniques et les témoins de références ont une émergence assez faible comparés aux plus faibles doses et au témoin négatif qui a enregistrés respectivement 96,4 et $95,12 \%$ du taux de chrysalide et l'émergences des adultes. Les doses d'application de 100, 75,50,25 et $10 \%$ des pesticides botaniques ont enregistré $100 \%$ de mortalité pendant 9 jours sur les larves L2. Ces résultats confirment l'idée (Thomas et al., 1997), vu que l'insecte déjà infecté n' arrive plus à s'alimenter correctement et meurt. D'une manière générale, à l'émergence, des femelles sont plus nombreuses que les males. Ce résultat est contraire à celui obtenu par Douro Kpindou (2011) suite au contrôle des larves de 3ème et de 4ème stade de $H$. armigera par les champignons entomopathogènes Beauveria bassiana et Metarhizium anisopliae par contre ce taux élevé de femelles émergées est similaire à celui observé par Surekha Devi (2009) suite à l'exposition de larves de $H$. armigera à une souche de Bacillus thuringiensis. En effet, la présence des pesticides botaniques a réduit significativement la consommation des feuilles par les larves. Les surfaces de feuilles de chou traitées aux différents extraits d'huile végétales consommées par les larves de P. xylostella furent globalement plus faibles que sur les feuilles témoin. Plus la concentration est élevée, plus la feuille est inappétence pour les larves. Dans une étude effectuée par Anam et al. (2006) une réduction significative des surfaces de feuilles de niébé traitées avec différentes concentrations d'huile de neem $(0,25 ; 0,5 ; 1$ ; 2 ; et 4\%) consommé par les larves de Epilachna dodecastigma Wied a été constatée en comparaison au témoin. À la suite d'un traitement de $24 \mathrm{~h} ; 28,880 \mathrm{~mm} 2$ de feuilles ont été consommées contre $40,101 \mathrm{~mm} 2$ pour le témoin. Les effets anti-appétants observés 
sont en fait fortement corrélés avec les réponses sensorielles des chémorécepteurs sur les pièces buccales des insectes (Mordue (Luntz) et al. 1998). De la position de ces récepteurs (tarses, pièces buccales, cavité buccale) dépend le comportement alimentaire de l'insecte. Mais outre la dissuasion alimentaire par suite de l'inappétence des feuilles traitées, la mortalité et la chrysalidation des larves sont liées à des perturbations des mues. Les effets sur la mue sont dus à des perturbations dans la synthèse et la libération des ecdystéroïdes (hormones de la mue) par inhibition de la libération de l'hormone provenant des glandes prothoraciques (Mordue (Luntz) et Nisbet, 2000). Chez des insectes ayant reçu par injection de l'azadirachtine avant la production d'hormone, on a noté le blocage total de l'ecdysteroïde (Mordue (Luntz) et Nisbet, 2000). Cela a pour conséquence l'inhibition du développement de l'insecte puis de la mue larvaire et a entrainé la mort.

En ce qui concerne l'efficacité dans le temps des pesticides utilisés pour le contrôle des larves de P. xylostella, quelle que soit la durée d'exposition, les DL50 obtenues sont plus faibles respectivement pour le produit chimique de synthèse Lambda cyalothrine, Topbio, T. purpurea, CNSL, R. communis et T. neriifolia. Au début des tests, les pesticides ont eu une action répulsive sur $P$. xylostella. En effet, les larves ont quitté les feuilles traitées avec les pesticides. En dehors de cette action répulsive, l'étude des effets de ces pesticides ont montré chez P. xylostella des impacts sur le développement, la mue et la reproduction. En comparaison au témoin, les taux de mortalités élevés ont été relevés au niveau des populations de Plutella nourris sur des feuilles traitées avec Lambda cyalothrine et les pesticides botaniques. La dose de $1 \%$ des pesticides a augmenté, la mortalité chez $P$. xylostella en comparaison au témoin. Des études similaires avaient été précédemment fait sur les pucerons par Lowery et al. (1993), qui ont montré qu'une dose de $1 \%$ d'huile de neem a permis une réduction significative de la population du puceron en comparaison au contrôle. Les pesticides botaniques testés ont régulé la croissance de $P$. xylostella. En effet, une perturbation de la mue chez l'insecte a été observée chez des individus nourris sur des feuilles traitées. Cette perturbation chez les larves semble être due au blocage de la sécrétion des hormones de la mue. Ce blocage entraine l'arrêt du développement morphogénétique de la larve qui de ce fait, ne peut pas atteindre le stade adulte.

Les coefficients de régression positifs pour les deux pesticides indépendamment des ravageurs prédisent une augmentation de la mortalité des ravageurs dans le temps. Quel que soit le pesticide chimique de synthèse ou botanique, la comparaison des DL50 temporelles obtenues montre que la quantité de pesticides nécessaire à l'élimination les larves du stade 2 est inférieure à celle requise pour éliminer les larves du troisième stade $P$. xylostella. La DL50 de T. purpurea nécessaire pour contrôler la moitié des larves du troisième stade 48 heures après traitement est de 13,4\% d'huile de $T$. purpurea contre $8,1 \%$ pour contrôler les larves du stade 2. Cette forte différence pourrait être due au poids de chaque insecte. En effet, de façon globale, la dose létale s'exprime généralement en unité de substance par unité de masse. Les larves de 3ème stade de $P$. xylostella étant plus grosse que le deuxième stade, il faudrait alors plus de substance pour les éliminer. Ce constat a été fait par Bateman et al. (1996) qui ont trouvé que l'infection des criquets par les champignons entomopathogènes est fonction de leur poids. De même, les travaux de Douro Kpindou (2011) ont révélé qu'il faut une dose de Metarhizium anisopliae plus élevée pour contrôler les larves de 4ème stade de $H$. armigera par rapport aux larves de 3ème stade. A ce facteur lié à la masse pourrait également s'ajouter le mode d'alimentation de l'insecte. Les pesticides biologiques utilisés peuvent être utilisés en milieu réel pour déterminer la dose d'application pouvant remplacer le l'insecticide chimique de synthèse. 


\section{CONCLUSION}

En conclusion nous pouvons retenir que les différentes huiles végétales et le baume de cajou extraction à froid testés ont régulé la croissance de la population de $P$. xylostella utilisées en augmentant la mortalité des larves réduisant ainsi le taux d'adultes émergés. Les résultats obtenus après les tests biologiques réalisés ont révélé l'effet dose-réponse entre les larves traitées et les doses des pesticides utilisées. De même, le temps d'exposition influence la dose de pesticide à utiliser. Pour chaque pesticide, aussi bien les concentrations et le temps d'exposition ont montré des effets significatifs utiles pour la gestion et le développement de P. xylostella. Une analyse comparative des pesticides botaniques a montré une efficacité plus élevée respectivement de Topbio, de l'huile de Tephrosia, du CNSL à froid, de l'huile de Ricinus et de Thevetia. En perspective nous envisageons la caractérisation chimique des différentes huiles végétales utilisées et proposé une formulation en vue de déterminer la dose d'application optimale de ces pesticides botaniques en milieu réel.

\section{REMERCIEMENT}

Nous remercions l'Agence Universitaire de la Francophonie (AUF), pour leur aide financière à travers le Projet de Recherche Partenarial 2018. Nous remercions également l'entreprise Biophyto SARL pour sa contribution au projet.

\section{RÉFÉRENCES}

Abbott, W.S. 1925. A method of computing the effectiveness of an insecticide. Journal of Economic Entomology 18(2):265-267.

Agboka, K., Agbodzavu, K.M., Tamò, M. and Vidal, S. 2009. Effects of plant extracts and oil emulsions on the maize cob borer Mussidia nigrivenella (Lepidoptera: Pyralidae) in laboratory and field experiments. International Journal of Tropical Insect Science 29:185-194.
Akpo, A. 2017. Evaluation de l'efficacité des extraits des Plantes locales pour le contrôle des vecteurs du paludisme résistant aux pyréthrinoïdes au Bénin (Afrique de l'Ouest). Thèse de Doctorat de l'Université d'Abomey-Calavi. 253p.

Anam, M., Ahmad, M. and Haque, M.A. 2006. Efficacy of neem oil on the biology and food consumption of Epilachna beetle, Epilachna dodecastigma (Wied.). J Agric Rural Dev. 4(1\&2), 83-88 http://www. cabdirect.org/search.htmlq= au\%3A\%22Shobhat.

Anani, B.Y., Gounou, S., Chabi-Olaye, A., Smith, H. and Schulthess, F. 2004. The effect of neem (Azadirachta indica A. Juss) oil on oviposition, development and reproductive potentials of Sesamia calamistis Hampson (Lepidoptera: Noctuidae) and Eldana saccharina Walker (Lepidoptera: Pyralidae). The Royal Entomological Society, Agricult. Forest Entomol. 6:223-232.

Aouinty, B., Oufara ,S., Mellouki, F.and Mahari, S. 2006. Evaluation préliminaire de l'activité larvicide des extraits aqueux des feuilles du ricin (Ricinus communis L.) et du bois de thuya (Tetraclinis articulata (Vahl) Mast.) sur les larves de quatre moustiques culicidés: Culex pipiens (Linné), Aedes caspius (Pallas), Culiseta longiareolata (Aitken) et Anopheles maculipennis (Meigen). Biotechnology, Agronomy, Society and Environment 10: 67.

Argenti, O. 2010. Approvisionnement et distribution des aliments dans les villes. Symposium international : Horticulture urbaine et périurbaine au siècle des villes. Symposium international Dakar, République du Sénégal, 6-9 décembre $2010: 30$.

Azonkpin, S., Chougourou, D., Bokonon, A., Dossou, J. and Ahonton, L. 2019. Efficacité du baume de cajou contre les chenilles carpophages du Cotonnier au Nord Du Benin. European Scientific Journal 14(24).

Bateman, R., Carey, M., Batt, D., Prior, C., Abraham, Y., Moora, D., Jenkinsm N. and 
Fenlon, J. 1996. Screening for virulent isolates of Entomopathogenic fungi against the desert locust, Shistocerca gregaria (ForskAl). Biocont. Sc. and Technol 6:549 - 560.

Chilcutt, C.F. and Tabashnik, B.E. 1997. Hostmediated competition between the pathogen Bacillus thuringiensis and the parasitoid Cotesia plutellae of the diamondback moth (Lepidoptera: Plutellidae). Environmental Entomology 26(1):38-45. https://doi.org/ 10.1093/ee/26.1.38.

Chougourou, C.D., Dellouh, P.L., Agbaka, A., N'guessan, K.R. and Gbenou, J.D. 2012. Toxicité et effets répulsifs de certaines huiles extraites des plantes locales béninoises sur la mouche domestique Musca domestica L. (Diptera Muscidae). Journal of Applied Biosciences 55:39533961.

Douro Kpindou, O.K. 2011. Potentialités des entomopathogènes Metarhizium anisopliae (Hypocreales: Clavicipitaceae) et Beauveria bassiana (Hypocreales: Ophiocordycipitaceae) dans la gestion de Helicoverpa armigera (Hûbner) (Lepidoptera: Noctuidae) au Bénin. Thèse de doctorat Univ de Lomé Togo. 186pp.

Dovlo K.A. 2007. Quelques aspects socioculturels et écologiques de l'utilisation des pesticides dans la production maraîchère de la zone portuaire de Lomé. Mémoire de D.E.A., Université de Lomé, $58 \mathrm{pp}$.

FAO. 2012. La sous-alimentation dans le monde en 2012. www.fao.org/docrep/ 17/ i3027f/i3027f02.pdf

FAOSTAT. 2013. (http:// faostat.org). Food and Agriculture Organisation, United Nation.

Furlong, M.J., Wright, J. and Dosdall, L.M. 2012. Diamondback moth ecology and management: Problems, progress and prospects. Annual Review Entomology 58: 517-41.

Garima, Z. and Amla, B. 2011. Thevetia peruviana (Pers.) Schum: A plant with enormous therapeutic potential. Journal of Pharmacy Research 4(12):4461-4464.

Glitho, I.A., Ketoh, G.K., Nuto, P.Y., Amevoin, S.K. and Huignard, J. 2008. Approches non toxiques et non polluantes pour le contrôle des populations d'insectes nuisibles en Afrique du Centre et de l'Ouest. In: Biopesticides d'origine végétale (2è édition), Regnault R, Philogène BJR, Vincent C (eds). Editeur Tec et Doc / Lavoisier : 207-217.

Guilloux, T. 2000. Etude de la variabilité biologique, biochimique et génétique de populations d'origines géographiques différentes de Cotesia plutellae (Kurdjumov) (Hymenoptera Braconidae), parasitoide de la teigne des Brassicacées Plutella xylostella (L.) (Lepidoptera : Yponomeutidae). Thèse de Doctorat d'université, Université Paul.

Gupta, P.D. and Thorsteinson, A.J. 1998. Food plant relationship of diamondback moth (Plutella 171 maculipennis Curt.) II. Sensory relationship ofd ovoposition of the adult female. Entomol. Exp. Appl 3:305314. Université Paul Valery, Montpellier III. $215 \mathrm{p}$.

Hill, T.A. and Foster, R.E. 2000. Effect of insecticides on the diamondback moth (Lepidoptera: Plutellidae) and its parasitoid Diadegma insulare (Hymenoptera: Ichneumonidae). J. Econ. Entomol., 93(3): 763-768.

Hooks, C.R.R. and Johnson, M.W. 2003. Impact of agricultural diversification on the insect community of cruciferous crops. Crop Protection 22:223-238.

Huang, Z., Ali, S., Ren, S.X. and Wu, J.H. 2010. Effect of Isaria fumosoroseus on mortality and fecundity of Bemisia tabaci and Plutella xylostella. Insect Science and its Application 17:140-148.

James, B., Atcha, C., Godonou, I. and Baimey, H. 2005. Summary of activities and achievements, 2003-2005. Healthy vegetables through participatory IPM in peri-urban areas of Benin, International 
Institute of Tropical Agriculture (IITA). $49 \mathrm{pp}$.

James, B., Atcha-Ahowe, C., Godonou, I., Baimey, H., Goergen, G., Sikirou, R., Toko, M. 2010. Gestion intégrée des nuisibles en production maraîchère : Guide pour les agents de vulgarisation en Afrique de l'Ouest. Institut international d'agriculture tropicale (IITA), Ibadan, Nigeria. p. 120.

Kétho G.K., Ghlitho, A.I. and Huignard, J. 2002. Susceptibility of the bruchid Callosobruchus maculatus (Coleoptera: Pteromalidae) and its parasitoid Dinarmus basalis (Hymenoptera: Petromalidae) to three essential oils. Journal of Economic Entomology 95:174-182.

Kumar, O., Lakshmana, Rao, P.V., Pradhan, S., Jayaraj, R., Bhaskar, A.S., Nashikkar, A.B. and Vijayaraghavan, R. 2007. Dose dependent effect of ricin on DNA damage and antioxidant enzymes in mice. Cell and Molecular Biology (Noisy.-le-grand) 53: 92-102.

Laghdaf, T. and Ferji, Z. 2005 : Evalution de quleques plantes nématicides et produits. Nationle sur les Substences Naturelles : La valorisation et dévloppement, Errachidia, Marco. pp. 29-30.

Liu, S.S., Wang, X.G., Guo, S.J., He, J.H. and Song, H.M. 1995. A survey of insect parasitoids of Plutella xylostella and the seasonal abundance of the major parasitoids in Hangzhou, China. In: Sivapragasan, A., Loke, W.H., Hussan, A.K. and Lim, G.S. (Eds.) The management of diamondback moth and other crucifer pests. pp. 61-66. In: Proceedings of the Third International Workshop. Mardi, Kuala Lumpur, Malaysia.

Lowery, D.T., Isman, M.B. and Bhard, N.L. 1993. Laboratory and field evaluation of neem for the control of aphids (Homoptera:Aphididae). J. Econ. Entomol., 6:864-870.

Macharia, I., Löhr, B. and De Groote, H. 2005. Assessing the potential impact of biological control of Plutella xylostella (diamondback moth) in cabbage production in Kenya. Crop Protection 24:981-989.

Maddox, J.V. 1982. Use of insect pathogens in pest management. pp. 175-216. In: Metcalf, R.L. and Luckmann, W.H. (Eds.). Introduction to insect pest management. 2nd ed. New York, USA: Wiley.

Mayunga, H.H.N. 2002. Natural chemicals for disease and insect management; professorial inaugural lecture, University of Dar es salaam, Tanzania. pp. 130-134.

Mollah, J.U. and Islam, W. 2007: Toxicity of Thevetia peruviana (Pers) Schum. Extract to adults of Callosobruchus maculatus $\mathrm{F}$. (Coleoptera: Bruchidae). Agric. Rural Dev. 5(1 \& 2):105-109.

Mondedji, A.D. 2010. Potentiel d'utilisation d'extraits de feuilles de Neem (Azadirachta indica A. Juss) et de papayer (Carica papaya $\mathrm{L}$.) dans le contrôle des insectes ravageurs du chou (Brassica oleracea $\mathrm{L}$.) en zones urbaines et périurbaines au sud du Togo. Thèse de doctorat, Université de Lomé, Togo. 195pp.

Mordue (Luntz), A. J. and Nisbet, A. J. 2000. Azadirachtin from the neem tree Azadirachta indica: its action against insects An. Soc. Entomol. Bras. 29(4): Londrina.

Mordue Luntz, A.J., Simmonds, M.S.J., Ley, S.V., Blaney, W.M., Mordue, W., Nasiruddin, M. and Nisbet, A.J. 1998. Actions of azadirachtin, a plant allelochemical, against insects. Pestic. Sci. 54:277-284.

Olsnes, S., Refsnes, K. and Pihl, A. 2004. Mechanism of action of the toxic lectins abrin and ricin. Nat. 249:627-631.

Philogène, B.J.R., Regnault-Roger, C. and Vincent C. 2003. Produits phytosanitaires insecticides d'origine végétale: promesses d'hier et d'aujourd'hui. In: Biopesticides d'Origine Végétale, Roger $\mathrm{C}$, Philogène

Puyvelde, L.V., De Kimpe, N., Mudaheranwa, J.P., Gasiga, A. and Schamp, N. 2010. Journal of Natural Products. 50:349. 
Sanda, K., Koba, K., Poutouli, W., Idrissou, N. and Agbossou, A.B. 2006. Pesticidal properties of Cymbopogon schoenatus against the diamondback moth Plutella xylostella L. (Lepidoptera: Hyponomeutidae). Discovery and Innovation 19: 220-225.

Sarfraz, M., Keddie, A.B., Dosdall, L.M., 2005. Biological control of the diamondback moth, Plutella xylostella: A review Published online 20 July 2005. Biocontrol Sci. Techn. 15:763-789.

Shelton, A.M., Roush, R.T., Wang, P. and Zhao, J.Z. 2007. Resistance to insect pathogens and strategies to manage resistance: An update. pp. 793-811. In: Field Manual of Techniques in Invertebrate Pathology. Kluwer Academic Press.

Slotkin, T.A., Stadler, A., Skavicus, S. and Seidler, F.J. 2016. Adolescents and adults differ in the immediate and long-term impact of nicotine administration and withdrawal on cardiac norepinephrine. Brain Res. Bull., 122:71-75.

Surekha Devi, V. 2009. Interaction of acid exudates in chickpea with biological activity or cry toxins from Bacillus thuringiensis berliner against Helicoverpa armigera (Hubner). PhD thesis, Acharya N G Ranga Agricultural University.

Tabashnik, B.F. and Mau, R.F.L. 1990. Suppression of diamondback moth (Lepidoptera: Plutellidae) oviposition by overhead irrigation. J. Econ. Entomol., 79: 189-191.

Thomas, M.B., Blanford, S. and Lomer, C.J. 1997. Reduction of feeding by the variegated grasshopper, Zonocerus variegatus, following infection by the fungal pathogen, Metarhizium flavoviride. Biocontrol Sci. Technol., 7:327-334.
Tounou, A.K., Mawussi, G., Amadou, S., Agboka, K., Gumedzoe, Y.M.D. and Sanda, K. 2011. Bio-insecticidal effects of plant extracts and oil emulsions of Ricinus communis L. (Malpighiales: Euphorbiaceae) on the diamondback, Plutella xylostella L. (Lepidoptera: Plutellidae) under laboratory and semi-field conditions. J. Appl. Biosci., 43:2899-2914.

Touqeer, S., Saeed, M.A. and. Ajaib, M.A. 2013. Review on the phytochemistry and pharmacology of Genus Tephrosia. Phytopharmacology 4:598-637.

Verkerk, R.H.J. and Wright, D.J. 1996. Multitrophic interactions and management of the diamondback moth: A review. Bulletin Entomological Research 86:205216.

Wainwright, H., Wanyamay, C. and Cherotich, N. 2013. Biopesticides and their commercialisation in Africa. pp. 189-191. In: Proceedings of the First International Conference on Pesticidal Plants, 21-24 January 2013, Egerton University and ICIPE, Nairobi, Kenya.

Wanyika, H.N., Kareru, P.G., Keriko, J.M., Gachanja, A.N, Kenji, G.M. and Mukiira, N.J. 2009. Contact toxicity of some fixed plant oils and stabilized natural pyrethrum extracts against adult maize weevils (Sitophilus zeamais Motschulsky). African Journal of Pharmacy and Pharmacology 3(2):066-069.

Warwick, S.I., Francis, A. and Mulligan, G.A. 2003. Brassicaceae of Canada. Government of Canada. Available: http://www.cbif.gc. ca/spp_pages/brass/index_e.php. Biocontrol Sci. Technol. pp. 401-425.

Williamson, O.E. 2002. The theory of the firm as governance structure: From choice to contract. Journal of Economic Perspectives 16(3). http://groups.haas. berkeley.edu/bpp/oew/choicetocontract.p df 